\title{
INCURSÕES, DOENÇAS NEGLIGENCIADAS E A PROMOÇÃO DA SAÚdE NO PROGRAMA SAÚDE NA ESCOLA (PSE): A VISÃO DOS GESTORES LOCAIS
}

\author{
INCURSIONS, NEGLECTED DISEASES AND THE PROMOTION OF \\ HEALTH IN THE SCHOOL HEALTH PROGRAM (PSE): THE VISION \\ OF LOCAL MANAGERS
}

\author{
Sheila Soares de Assis $^{1}$ \\ Tania Cremonini Araujo-Jorge ${ }^{2}$
}

RESUMO: Introdução: Caracterizado como uma estratégia intersetorial oriunda da articulação entre os Ministérios da Saúde e da Educação, o Programa Saúde na Escola (PSE) possui como suas premissas a promoção da saúde, o controle, prevenção e detecção de agravos. Em contrapartida, as doenças negligenciadas afetam a vida de cerca de 1 bilhão de pessoas em todo o mundo e tem exigindo esforços integrados para sua superação. Assim, o PSE possui o potencial de contribuição para a diminuição deste cenário. Objetivo: analisar a visão dos representantes da gestão municipal do PSE sobre a estrutura do programa, a promoção da saúde e a abordagem das doenças negligenciadas. Metodologia: Para tal fez uso de entrevistas semiestruturadas que foram examinadas por meio da análise de conteúdo, categorização temática. Ao todo, seis representantes da gestão municipal do PSE foram entrevistados. Resultados: Emergiram como pontos críticos a alternância de temas a serem trabalhados no PSE, verticalização do diálogo entre as três esferas governamentais, ausência de recursos educativos para abordagem nas práticas educativas e a necessidade de maior abordagem sobre a tuberculose, hanseníase e verminoses. A integração viabilizada pela intersetorialidade, a melhoria da assistência aos estudantes e estratégias educativas dialógicas se caracterizaram como aspectos resolutos dentro da proposta. Conclusão: Embora haja avanços em relação a uma abordagem mais holística sobre a saúde, permanece como desafio a promoção de diálogo entre os diferentes atores que constituem o programa, bem como a formação dos profissionais para a gestão e operacionalização do PSE. Além do mais, é necessário superar a ideia

\footnotetext{
${ }^{1}$ Pós Doutoranda do Programa de Pós Graduação em Ensino em Biociências e Saúde. Laboratório de Inovações em Terapia, Ensino e Bioprodutos (LITEB). Instituto Oswaldo Cruz / Fundação Oswaldo Cruz - RJ.

${ }^{2}$ Pesquisadora titular em saúde pública. Chefe do Laboratório de Inovações em Terapias, Ensino e Bioprodutos (LITEB). Instituto Oswaldo Cruz / Fundação Oswaldo Cruz - RJ.
} 
simplista de complementariedade entre os campos da saúde e educação e se fomente a integração de fato. Resultando, deste modo, que o setor da saúde não seja um mero executor de ações biomédicas no espaço escolar.

Palavras chave: Colaboração Intersetorial. Educação em Saúde. Integralidade em Saúde.

ABSTRACT: Introduction: Categorized as an intersectoral strategy arising from the articulation between the Ministries of Health and Education, the Health at School Program (HSP) has as its premises the promotion of health, the control, prevention and detection of diseases. In contrast, neglected diseases affect the lives of nearly 1 billion people worldwide and have required integrated efforts to overcome them. Thus, the HSP has the potential to contribute to reducing this scenario. Objective: to analyze the view of the HSP municipal managementrepresentatives on the program structure, health promotion and the approach to neglected diseases. Methodology: For such, it made use of semi-structured interviews that were examined through content analysis, thematic categorization. In all, six representatives of the HSP municipal management were interviewed. Results: The alternation of themes to be worked on in the HSP, vertical dialogue between the three governmental spheres, lack of educational resources to address educational practices and the need for a greater approach to tuberculosis, leprosy and worms emerged as critical points. The integration made possible by intersectoriality, the improvement of assistance to students anddialogical educational strategies were characterized as resolute aspects within the proposal. Conclusion: Although there are advances in relation to a more holistic approach to health, the challenge remains to promote dialogue between the different actors that make up the program, as well as the training of professionals for the management and operationalization of the PSE. Furthermore, it is necessary to overcome the simplistic idea of complementarity between the fields of health and education and to promote de facto integration. As a result, the health sector is not a mere executor of biomedical actions in the school space.

Keywords: Intersectoral collaboration. Health education. Integrality in Health. 


\section{INTRODUÇÃO}

O Programa Saúde na Escola (PSE) é uma estratégia intersetorial iniciada em 2007, sendo fruto da parceria entre o Ministério da Saúde e da Educação. Dentre as finalidades do PSE está o aprofundamento dos objetivos do SUS voltados para a consolidação de um sistema de saúde universal, equânime e integral, concretizando as ações de promoção da saúde e da intersetorialidade (BRASIL, 2007). Na prática, as equipes da Estratégia de Saúde da Família (ESF) ficam responsáveis pelas escolas vinculadas ao programa e que se encontram em sua área de abrangência. As equipes de saúde são responsáveis por realizar monitoramento, avaliação das condições de saúde dos alunos, bem como o desenvolvimento de atividades educativas em conjunto com a equipe de profissionais da escola (BRASIL, 2007). As equipes de saúde devem ainda participar da construção do Plano Político Pedagógico (PPP) das escolas no que se refere aos temas relacionados à saúde e estes devemconsiderar a realidade social e escolar dos alunos (BRASIL, 2007).

As ações relacionadas às doenças negligenciadas no âmbito do PSE foram indicadas pela primeira vez no manual do programa de 2011(BRASIL, 2011). Nos anos subsequentes, essa questão alternou sua presença nos documentos norteadores do programa e vez ou outraum agravo foi eleito e passou a figurar como ponto a ser abordado nas atividades desencadeadas. Por exemplo, o Manual do Gestor destinado aos anos de 2019 a 2020 indicou, dentre as ações, prioritárias as "ações de combate ao mosquito Aedes aegypti" (BRASIL, 2019, p. 2). Contudo, como aborda Lopes; Nogueira; Rocha (2018) os estudos que buscam a compreensão do PSE têm exposto que o programa possui uma abordagem que contempla muito mais os aspectos relacionados à prevenção de doenças do que às diferentes determinações sociais da saúde (ROCHA; DAVID, 2015). As doenças negligenciadas representam um desafio à saúde global com cerca de 1 bilhão de pessoas afetadas no mundopor pelo menos um dos treze agravos prioritários (WHO, 2017). Portanto, o objetivo do presente estudo é analisar a visão dos representantes 
da gestão municipal do PSE sobre a estrutura do programa, a promoção da saúde e a abordagem das doenças negligenciadas.

\section{MÉTODO}

\section{Características da pesquisa, delimitação do estudo e aspectos éticos}

Delimitou-se como território para o estudo os municípios de Rio Branco (AC) e Rio de Janeiro (RJ). O projeto de pesquisa foi aprovado pelo Comitê de Ética em Pesquisas (CEP/IOC-FIOCRUZ) sob o número de protocolo 114.584. O estudo se caracteriza como qualitativo. Os dados foram coletados por meio de entrevistas semiestruturadas. Essa técnica foi escolhida em detrimento de outras por ser um processo dinâmico em que a principal finalidade é coletar informações de interesse ao estudo em execução e esclarecer como os elementos sobre aquele tópico estão narrativamente construídos (BRITTEN, 2005).

O roteiro utilizado foi subdividido em dois eixos: 1) Políticas públicas e o PSE e; 2) Situação local e as doenças negligenciadas. As questões abordadas foram elaboradas após a análise de documentos de referência do PSE e de doenças negligenciadas. $O$ instrumento foi validado previamente com uma subamostra de indivíduos que compõe ao grupo de investigados. As entrevistas foram realizadas individualmente e tiveram duração de 35 minutos a 1 hora e 45 minutos. Ao todo, seis profissionais participaram da pesquisa, todos participantes do Grupo de Trabalho Intersetorial (GTI) municipal que coordena o PSE.

Para exame dos dados, optou-se pela técnica de análise de conteúdo, categorização temática (BARDIN, 2009). Essa modalidade de análise é estruturada em três etapas, a saber: 1) pró-análise; 2) exploração do material e; 3) tratamento dos resultados e interpretação. A primeira fase consiste em uma organização inicial dos dados composto por uma leitura flutuante e escolha do material a ser trabalhado. Já a segunda etapa é o desenvolvimento das categorias de análise e identificação 
das unidades correspondentes. Por fim, a terceira fase compreende a inferência e interpretação dos resultados. Exige-se nessa etapa a análise reflexiva e crítica (BARDIN, 2009). A após a realização da segunda etapa de análise foram estabelecidas três categorias (quadro 1):

Quadro 1: Categorias de análise das entrevistas e aspectos abordados.

\begin{tabular}{|l|l|}
\hline \multicolumn{1}{|c|}{ CATEGORIA } & \multicolumn{1}{c|}{ ASPECTOS ABORDADOS } \\
\hline $\begin{array}{l}\text { Estrutura } \\
\text { funcionamento do PSE }\end{array}$ & $\begin{array}{l}\text { Questões técnicas sobre a estrutura e a dinâmica de } \\
\text { funcionamento do programa, bem como definição da agenda } \\
\text { de prioridades do programa. }\end{array}$ \\
\hline $\begin{array}{l}\text { Recursos humanos, Definição e descrição dos recursos humanos envolvidos no } \\
\text { materiais, financeiros efuncionamento do programa, suporte material, financeiro e } \\
\text { processos formativos }\end{array}$ & formativo disponibilizado para a operacionalização do PSE. \\
\hline $\begin{array}{l}\text { Promoção da Saúde, Refere-se as percepções creditadas a promoção da saúde } \\
\text { as doençasnas ações desenvolvidas pelo PSE e o lugar ocupado pelas } \\
\text { negligenciadas e outros doenças negligenciadas nas pautas do programa, bem como } \\
\text { temas abordados }\end{array}$ & \begin{tabular}{l} 
outras situações de saúde ou agravos citados. \\
\hline
\end{tabular}
\end{tabular}

Fonte: Produzido pelos autores.

\section{RESULTADOS E DISCUSSÃO}

Perfil dos entrevistados, contexto das entrevistas e apresentação dos resultados

As características dos profissionais entrevistados são sumarizadas no quadro 2. 
Quadro 2: Características dos representantes da gestão municipal entrevistados.

\begin{tabular}{|c|l|c|c|}
\hline Gestor & \multicolumn{1}{|c|}{ Escolaridade } & Idade & $\begin{array}{c}\text { Tempo de } \\
\text { atuação na } \\
\text { gestão do PSE }\end{array}$ \\
\hline Gestor 1 & $\begin{array}{l}\text { Especialista em Promoção da Saúde. } \\
\text { Graduada em geografia epedagogia. }\end{array}$ & 34 anos & 2 anos. \\
\hline Gestor 2 & $\begin{array}{l}\text { Graduada em medicina, com } \\
\text { especialização em pediatria. }\end{array}$ & 51 anos & 8 anos. \\
\hline Gestor 3 & $\begin{array}{l}\text { Mestre em Ciências, especialista em } \\
\text { Informação e Comunicação em Saúde e } \\
\text { pediatria. Graduada emmedicina }\end{array}$ & 65 anos & 3 anos \\
\hline Gestor 4 & $\begin{array}{l}\text { Mestre em Saúde da Família e graduada } \\
\text { emPsicologia. }\end{array}$ & 62 anos & 2 anos \\
\hline Gestor 5 & $\begin{array}{l}\text { Mestre e graduada em Serviço Social. } \\
\text { Especialista em SaúdePública. }\end{array}$ & 54 anos & 7 meses \\
\hline Gestor 6 & Graduada em pedagogia & 52 anos & 1 ano \\
\hline
\end{tabular}

Fonte: Produzido pelos autores.

Os profissionais vinculados as Secretárias Estaduais de Saúde pertencem a setores relacionados à promoção da saúde. O que denota uma preocupação marcada em relação ao PSE ao assimilar o programa com um viés mais holístico sobre a saúde. Contudo, em Rio Branco, a Secretária Estadual de Saúde, no período de realização das entrevistas, era dividida em dois prédios e as equipes eram alocadas em um ou outro edifício. Tal divisão dificulta ainteração entre os diferentes profissionais, principalmente no que se refere à equipe do PSEe a de vigilância em saúde. Já no Rio de Janeiro, a possibilidade de diálogo é facilitada entre os profissionais que são gestores do PSE e outros possíveis colaboradores devido ao espaçofísico que ocupam.

Questões sobre a estrutura e funcionamento do PSE, bem como pontos relevantes em relação a intersetorialidade serão explorados nas seções subsequentes. A seguir serãodiscutidos os resultados das três categorias de análise dos dados obtidos a partir da interlocução com os profissionais. 


\section{Estrutura e funcionamento do PSE}

Há diferenças em relação a estrutura de gestão do PSE entre o município do Rio de Janeiro e de Rio Branco. Enquanto que o PSE de Rio Branco o Grupo de Trabalho Intersetorial (GTI) é composto por membros da secretaria de saúde e de educação, o GTI dacidade do Rio de Janeiro engloba também um gestor da secretária de desenvolvimento social. Essa formatação onde se conta com atores de diferentes núcleos de atuação atende a uma demanda indicada nos documentos de referência do PSE.

Recomenda-se que os GTls englobem obrigatoriamente profissionais das secretarias de saúde e educação. Enquanto que os profissionais de outras secretarias apresentam participação facultativa, mas que atendam as demandas locais de forma coletiva (BRASIL, 2011). Garantida na constituição, a saúde transcende a um único setor e é assegurada como um direito universal e se caracteriza como dever do Estado fornecer subsídios para sua oferta, sejam esses econômicos, sociais, ambientais e advindos de outras esferas (COSTA et al., 2009; MATTOS, 2009). Portanto, o PSE caminha para uma prática de materialização deum dispositivo constitucional.

Em relação a ação do PSE, no relato dos gestores, elas aparecem como inconstantes e como um fator que dificulta o trabalho da gestão e dos atores incumbidos da execução dasações do programa. Tal questão é referenciada na fala do gestor 1:

"Com o PSE a gente vem aprendendo a trabalhar a cada ano, porque mudam as diretrizes. Um item que é essencial em um e no outro ano ele sai e eles vão acrescentando novas diretrizes de acordo com que eles vão achando necessário. (...) às vezes a gente ainda está encaminhando num processo aí muda e a gente tem que fazer outro direcionamento" (Gestor 1).

A referida alternância deve-se ao destaque atribuído a determinados temas nos documentos que norteiam o programa. Essa mudança abrupta é interpretada 
como um fator negativo entre os representantes entrevistados, pois exige uma reestruturação da atuação da equipe. Por outro lado, tradicionalmente o setor da saúde tem sido responsabilizado quando há grandes demandas de atendimento às doenças ou epidemias. Exigindo, assim, uma resposta relativamente rápida (LEFEVRE e LEFEVRE, 2004). O PSE, como uma política que viabiliza o acesso da comunidade escolar aos serviços de saúde, torna-se uma estratégia importante nesse processo de atendimento a demanda embora na prática, para os atores envolvidos, possa ser algo conflituoso a se lidar.

Dentre os objetivos do PSE é descrito:

(...) articular as ações do Sistema Único de Saúde - SUS às ações das redes de educação básica pública, de forma a ampliar o alcance e o impacto de suas ações relativas aos estudantes e suas famílias, otimizando a utilização dos espaços, equipamentos e recursos disponíveis (BRASIL, 2007).

De acordo com os relatos dos gestores essa orientação tem sido alcançada. Quando indagados sobre os pontos positivos do PSE, todos destacaram a ampliação do acesso e a melhoria da qualidade da assistência à saúde destinado aos estudantes.

Além disso, a integração promovida pela proposta intersetorial do programa destacou-se não somente pelo caráter de prevenção de agravos, mas também em um melhor reconhecimento do território e um atendimento a promoção da saúde como exemplificado nos relatos:

"A intersetorialidade, a gente tem que integrar políticas públicas. Então, a saúde, educação e assistência, esse tripé ele tem funcionado bem dentro do PSE e agente agora tenta integrar também outros programas e ações que essas secretarias desenvolvem, mas de uma forma isolada, então tudo agora tem já um espaço no PSE, então vamos para esse espaço intersetorial" (Gestor 5).

“(...) eu acho que o destaque é a gente conseguir trabalhar intersetorialmente, eu acho que a gente está avançando, porque issoé muito difícil. Então, eu acho que a gente está conseguindo ter uma boa relação com a 
Secretaria de Educação, com a secretaria de Desenvolvimento Social, com a Casa Civil que agora entra e passa a ter um espaço respeitoso, de poder ser transparente, de poder dizer o que acha, eu acho que a gente avançou bastante nisso, tem sido um ponto positivo" (Gestor 4).

A operacionalização do PSE é viabilizada por meio da de uma gestão compartilhadae intersetorial. A intersetorialidade pressupõe em diálogo, mesmo que em alguns momentos hajam embates de ideias devido a formação e origem diversificada dos profissionais, mas isso tem sido superado no cotidiano do programa. A finalidade da intersetorialidade é responder de forma eficaz a solução ou compreensão de determinados problemas em sua plenitude (JUNQUEIRA, 2004).

Os profissionais sinalizam para uma sobrecarga dos profissionais de saúde que são responsabilizados unicamente pela saúde da população e indicam a necessidade de espaços de escuta para que se considerem os temas a serem abordados no PSE. Ou seja, na percepçãodo profissional há verticalização dos eixos do PSE e não acontece no programa um espaço de diálogo amplo.

Eu acho que a gente tem que escutar a própria população sobre quais são os problemas que são relevantes para aquele grupo, como é que está essas estatísticas [se refere a incidência de doenças no território], se a gente está tendo ações relevantes. $O$ que a gente não está valorizando? Mas isso não acontece, o que acontece é que você tem ali algumas coisas que você é obrigado a fazer. Tem que fazer isso e aquilo. Então, eu acho que os espaços de encontro com a população são muito rápidos" (Gestor 3 ).

É indicado também pelos gestores a falta de integração com outras esferas do Programa, principalmente, no âmbito federal Tal falta de integração reforça a questão da verticalização do programa.

Desde que eu cheguei no PSE do município a gente tem essa integração com a equipe daqui, mas eu mesma não tive nenhum contato com o PSE nacional direito. Eu não participei de nenhum movimento. Eu até gostaria, mas 
não tive a oportunidade de participar de algum movimento nacional sobre a estrutura do Saúde na Escola (Gestor 6).

Como verificado na fala dos entrevistados neste bloco, a alternância de temas que são enfatizados a cada ano, o período de adesão ao PSE, bem como a verticalização e ausência de diálogo são percebidos como conflituosos para a viabilização das ações do PSE e a atuação dos profissionais de saúde. Já a intersetorialidade, a melhoria da assistência ofertada aos estudantes e o viés da promoção da saúde dentro das atividades executadas se caracterizam como aspectos positivos do programa.

\section{Recursos humanos, materiais e processos formativos}

No relato dos entrevistados, é atribuído o papel que cabe a qual setor dentro das ações do PSE. Enquanto que o setor da saúde aparece como responsável pela elaboração e execução das ações, o setor da educação aparece como gerador de demandas a serem trabalhadas e agente mobilizador de público. Nesse sentido, 0 PSE, em sua prática, materializa uma perspectiva de intersetorialidade restrita (PAPOULA, 2006; LOPES; NOGUEIRA; ROCHA, 2018). Além disso, o relato dos gestores converge com a indicação de um protagonismo do setor da saúde no que se refere a estruturação do PSE (FERREIRAet al., 2012):

"Além das demandas já impostas pelo PSE nacional que tem no Ministério da Saúde e da Educação, a gente tem que atender... A gente quer atender as demandas do território. Então, tem muitas ações que não estão nos componentes do PSE, mas o território tem necessidade de agir. São ações solicitadas pelas escolas. Então, nósconstruímos ações” (Gestor 2).

"Primeiro a gente faz a visita na escola e às vezes a gente está levando outra coisa... A gente fala para a diretora, por exemplo: 'A gente está fazendo a antropometria das crianças'. Aí a diretorajá fala: 'Ah, vocês poderiam 
trazer outra coisa?' Aí eles já vão pedindo outra coisa que eles querem. A gente articula com a unidade de saúde porque quase tudo que a escola precisa de emergencial a gente tem como articular com a unidade de saúde." (Gestor 1).

“...) a escola tem uma agenda muito grande e às vezes também aquilo que a escola está te mandando não é aquilo que a saúde está oferecendo. Existem conflitos. Tem escolas que: 'Não, eu não quero isso agora. Eu estou precisando de outra coisa nesse momento"' (Gestor 4).

Em relação aos materiais educativos empregados nas ações do PSE quando disponibilizados acabam sendo repassados diretamente para o setor da saúde.

"Normalmente quando a saúde recebe algum material educativo ela passa para a gente passar para as escolas. Normalmente esse material, quando chega, vai direto para o pessoal da saúde. Quando a Secretaria Estadual manda ou o ministério manda alguma coisa, não manda direto para gente. Os materiais como panfletos, cartilhas... são mandados para a saúde e ela manda pra gente e a gente faz a distribuição de acordo... Mas desde que cheguei não recebemos nada" (Gestor 6).

Embora estes materiais sejam valorizados na prática educativa realizada por profissionais de saúde e professores no espaço escolar, a sua distribuição é deficiente (ASSIS, PIMENTA e SCHALL, 2013). Contudo, aponta-se também a controvérsia que envolve o uso desses recursos nas práticas de educação em saúde. Em trabalhos anteriores reportou-se o caráter verticalizado dos materiais educativos. Além disso, somam-se críticas por estes desconsiderarem a realidade a qual são utilizados, os atores envolvidos nas práticaseducativas e o uso constante de imagens e linguagem inadequadas (ASSIS, PIMENTA e SCHALL, 2013; KELLYSANTOS; MONTEIRO; ROSEMBERG, 2009; ROSEMBERG, SILVA; VASCONCELLOS-SILVA, 2002).

Também foi relatado a ausência de estratégias de formação específica direta para os gestores e/ou os atores envolvidos na realização das atividades do PSE. 
Assim, os próprios profissionais desenvolvem a estratégia de que são corresponsáveis pela formação doscolegas através da partilha de saberes técnicos.

"Mensalmente a gente se encontra, toda última terça feira do mês com os representantes da saúde, da educação e do desenvolvimento social. Todos os membros do GTI se reúnem a onde tem os informese alguém, normalmente da saúde, estuda um tema e apresenta para os demais" (Gestor 6).

"Nossa maior questão é essa de como é que a gente vai qualificar os nossos profissionais para atender mais e melhor, então esse é um desafio nosso, é um ponto desafiador" (Gestor 4).

A formação dos profissionais de saúde e educação envolvidos nas ações do PSE compreende ao componente III das diretrizes do PSE. Além da formação destes atores deveser considerado no contexto a construção de materiais didáticospedagógicos para serem empregados nas ações do programa (BRASIL, 2018). Além disso, os entrevistados também relataram a inexistência de formações específicas para atuação no PSE, seja a ofertadestinada aos gestores ou para os profissionais de saúde e educação que atuam diretamente no intermédio junto ao público escolar.

É imperativo que se tenha atenção a este fato, pois, na atualidade, a formação específica para os profissionais do PSE suprimido juntamente com a educação permanente em saúde proposta para os trabalhadores da saúde a fim de se construir um Sistema Único de Saúde (SUS) humanizado e alinhado com os fundamentos estabelecidos para seu funcionamento e consolidação. Assim, tendo em vista que o PSE almeja ampliar o acesso da comunidade escolar ao serviço de saúde, a formação dos profissionais envolvidos nessas ações não pode ser negligenciada.

Neste bloco destacou-se na fala dos gestores os papeis atribuídos ao setor da saúde e educação no âmbito do PSE. Foi denunciada a escassez de materiais educativos repassados as secretarias municipais para a execução das atividades educativas. Contudo, quando o repasse acontece, ele ocorre prioritariamente ao setor da saúde. Caracterizando, assim, o papel coadjuvante da área da educação 
dentro do PSE. Identificou-se também carência de processos que visem a formação específica para atuar no programa.

\section{Promoção da Saúde e as doenças negligenciadas}

A promoção da saúde objetivada no PSE remete diretamente a intersetorialidade. Segundo Junqueira (2004) este modelo de gestão intersetorial envolve a noção de integração do território, equidade e de direitos sociais. Embora ainda haja muitos desafios a serem enfrentados pelo PSE, no relato dos entrevistados indica-se o avanço do programa no sentido de se ocupar, atualmente, não somente com o diagnóstico, mas atuar no atendimento integraldos estudantes ao considerar a promoção da saúde e a prevenção de agravos.

"No passado eu acho que a saúde, fora do PSE, fazia muito mais diagnósticos, eu fazia, e colocava como relacionando à questão do fracasso ao sucesso escolar as questões de saúde e da doença. Então, eu acho que hoje a gente tem com enfoque mais na questão da prevenção, o PSE é mais a atuação na questão da prevenção e promoção à saúde, não na doença, isso tem mudado bastante com as ações intersetoriais. Então, eu penso que hoje mais do que o diagnóstico de doenças, a gente consegue fazer um trabalho, por exemplo, sobre as relações familiares dentro de uma escola, você está trabalhando a saúde emocional dessa criança, desse adolescente e de suas famílias. Então, a gente com essa visão ampliada a gente acaba melhorando as condições de saúde na questão da prevenção e da promoção, e não focando somente nos diagnósticos de doenças como o PSE em anos anteriores fazíamos levantamento de acuidade visual em todos os alunos. E o que se fazia com isso? (Gestor 5).

Essa atuação que privilegia também uma visão integral sobre a saúde da comunidade escolar. Segundo Buss et al. (2020) a promoção da saúde privilegia uma atuação participativa, multidimensional e se considera o ambiente do indivíduo em sua totalidade. Assim sendo, o PSE, segundo a fala dos entrevistados, parece 
atender a essa perspectiva. As práticas envolvendo a promoção da saúde são relativamente recentes quando comparadas aações de cunho curativo. A inclusão da perspectiva da promoção da saúde em programas deatenção a comunidade escolar como, por exemplo, o PSE representa um avanço no sentido de construção de um cenário mais equânime (BUSS et al., 2020; CASEMIRO, FONSECA e SECCO, 2014).

Essa visão é essencial quando tratamos das doenças negligenciadas. Embora o diagnóstico e o tratamento sejam elementos essenciais para que esses agravos sejam sanados, é imprescindível que considere o contexto a qual os indivíduos estão imersos. No Brasil, houveram avanços nos programas de controle de doenças infecciosas e parasitárias. Contudo, faz-se necessário o maior incentivo às ações que considerem as determinações sociais, culturais e os conhecimentos das populações afetadas (BARRETO et al., 2011).

Quando indagados sobre as doenças negligenciadas, os gestores reportam a emergência quanto a necessidade de abordagem da questão na pauta do PSE.

"Eu acho que a tuberculose precisa ter um investimento ainda em cima dela e a hanseníase idem. Tem uma coisa, por exemplo, que não existe mais... Ou seja, não se fala mais. Os vermes não existem mais. As parasitoses parecem que não existem mais. Quando você vai ver as estatísticas, milhões de pessoas no mundo ou trilhões estão com o parasita, mas toda vez que vai ver em texto base de referência isso não existe. Não se fala mais nisso. A gente tem a giárdia como um número expressivo em crianças e a doença vai alterando a vida delas, e percebe-se que não se dá muita importância, dá um remedinho e tal que passa. Não é nada efetivo. O tema nem é tratado como se deve" (Gestor 3).

"Há alguns meses uma escola ligou para a gente tirar urgente do banco daqui da secretaria algum profissional e enviar para lá, porque tinha uma professora com suspeita de estar com tuberculose. Aí eu acionei a equipe, acalmamos ela e a gente foi lá. Falamos que não é assim também e a gente fez um levantamento da carteira de vacinação das crianças também para saber se todos tomaram a BCG, as crianças que tinham contato com a professora. Fizemos esse levantamento, fizemos a 
atividade educativa com a escola toda e a professora nem estava com tuberculose. Então, ainda tem um tabu só com a suspeita" (Gestor 1).

Segundo a indicação contida na fala do entrevistado, a tuberculose e a hanseníase se caracterizam como agravos emergenciais a serem tratados. Em relação à tuberculose, Barreira (2018) indica o protagonismo do Brasil a nível mundial nas ações de controle da doença. Segundo o pesquisador, dentre as medidas essenciais para que se atinja a eliminação da tuberculose está o fortalecimento das ações intersetoriais. Em relação à hanseníase no Brasil, o Ministério da Saúde reportou que 28.660 que representa $92,6 \%$ do total dos casos nas Américas). Do total de casos diagnosticados no país, 1.705 (5,9\%) ocorreram em menores de 15 anos, ou seja, indivíduos em idade escolar (BRASIL, 2020). Portanto, o PSEse configura como um importante ator para a redução de agravos de importância no país.

Outra questão abordada na fala do entrevistado refere-se às geohelmintoses e outros parasitos associados à ausência de saneamento básico. Em 2013 e 2014 o Ministério da Saúde realizou ação integrada, realizada pelo para o controle de hanseníase, verminoses e tracoma. Ao longo das campanhas, administrou-se terapia medicamentosa preventiva para crianças e adolescentes em idade escolar (Brasil, 2016). No Brasil, a Pesquisa Nacional por Amostra em Domicílios (PNAD) indica que 68,3\% dos domicílios brasileiros possuem acesso à esgotamento sanitário (IBGE, 2020). Portanto, políticas baseadas em medidas isoladas de administração de medicamentos sem que haja um plano consistente visando a ampliação ao saneamento básico e estratégias educativas associadas a prevenção e o controledas verminoses torna-se falho.

Segundo os entrevistados, a realização de oficinas e a produção de materiais educativos próprios é estimulada pela escassez de repasse dos recursos prontos sobre temastratados no contexto do PSE.

"Em relação a hanseníase a gente até fez durante um tempo a produção de um material do próprio grupo. A construção foi junto com a gerência de dermatologia sanitária. A gente fez um material para ser levado para as escolas. É um livreto que depois a gente levou para 
várias escolas, testou e foi bem legal. Então, a gente tem esse material, mas no geral a gente, por exemplo, quando faz uma oficina, a gente constrói junto com as pessoas o material, a gente faz muita oficina, tem muita oficina interativa e a gente pega muitoo que as pessoas já sabem e trabalha em cima do que elas já sabem e de questões para o futuro." (Gestor 3).

As oficinas se constituem como espaços dialógicos de negociação de sentidos, discussão e interação. Constituem em ações não verticalizadas, mas de troca de saberes (SPINK; MENEGON; MEDRADO, 2014). Além disso, a produção de materiais educativos que os próprios profissionais de saúde farão uso em seu cotidiano remete ao processo de autoria coletiva na medida que a construção é compartilhada. Nesse sentido, o profissional de saúde não é apenas alguém que decora uma série de procedimentos e os repassa para a população, mas sim alguém que interage e caminha junto a ela em prol de ampliação da visão sobre determinado problema enfrentado (Vasconcelos, 2001).

Neste bloco discutiu-se a interface com a promoção da saúde a prática do PSE. Nos relatos dos entrevistados revelou-se a emergência da abordagem do tema das doenças negligenciadas. A realização de oficinas e a produção de materiais educativos próprios mostrou-se como importantes para promover o diálogo entre os diversos atores do programa, profissionais e escolares.

\section{CONCLUSÃO}

Compreender a visão dos representantes da gestão municipal do PSE sobre o programa, a promoção da saúde e as doenças negligenciadas é um esforço necessário para que sejam evidenciados como estes temas estão sendo apropriados a nível da gerência do programa. A partir das categorias de análise apresentadas foi possível verificar que a alternância de temas em destaque no programa, a verticalização, bem como a ausência de diálogo entre as esferas diferentes esferas do programa, ausência de formação específica para atuação no programa são questões que merecem atenção. 
Além disso, a tuberculose, a hanseníase e as verminoses associadas à ausência de saneamento básico foram consideradas as doenças negligenciadas que merecem mais atenção devido a sua abordagem inexistente ou insuficiente. Já a promoção da saúde parecedespontar nas práticas do programa atendendo, assim, a um de seus pressupostos. Há então uma mudança de paradigma, ainda que tímida, no que concerne as ações, historicamente consolidadas, de saúde no espaço escolar.

À guisa de concluir, reforçamos que o PSE como uma política intersetorial que agrega diferentes atores não pode se limitar a atribuir a estes profissionais um caráter simplista de complementariedade, mas é sim necessária uma integração que se processe de forma mais holística entre os campos da saúde e educação. Resultando, deste modo, que o setor da saúde não seja um mero executor de ações biomédicas no espaço escolar.

\section{REFERÊNCIAS BIBLIOGRÁFICAS}

ASSIS, S. S.; PIMENTA, D. N.; SCHALL, V. T. Materiais impressos sobre dengue: análise e percepções de profissionais de saúde e educação. Revista Brasileira de Pesquisa em Educação em Ciências, São Paulo, v. 13, n. 3, 2013.

BARRETO, M. L.; TEIXEIRA, M. G.; BASTOS, I. F.; XIMENES, A. A.; BARATA, R. B.; RODRIGUES, L. C. Sucessos e fracassos no controle de doenças infecciosas no Brasil: o contexto social e ambiental, políticas, intervenções e necessidades de pesquisa. The Lancet, 2011.

BRARREIRA, D. Os desafios para a eliminação da tuberculose no Brasil. Epidemiologia nos Serviços de Saúde, v. 27, n. 1, 2018.

BRASIL. Manual de adesão ao Programa Saúde na Escola. 2019 Disponível em: http://189.28.128.100/dab/docs/portaldab/documentos/pse/manual_adesao_2019_20.pdf.

Acesso em: 20 de fevereiro de 2021.

BRASIL. Ministério da Educação. Programa Saúde nas Escolas, 2018. Disponível em: http://portal.mec.gov.br/expansao-da-rede-federal/194-secretarias-112877938/secad- educacaocontinuada-223369541/14578-programa-saude-nas-escolas. Acesso em: 20 de fevereiro de 2021.

BRASIL. Ministério da Saúde. Secretaria de Atenção à Saúde. Departamento de Atenção Básica. Passo a passo PSE: Programa Saúde na Escola: tecendo caminhos da intersetorialidade / Ministério da Saúde. Secretaria de Atenção à Saúde. Departamento de Atenção Básica, Ministério da Educação. - Brasília: Ministério da Saúde, 2011.

BRASIL. Ministério da Saúde. Secretária de Vigilância em Saúde. Boletim Epidemiológico - 
Hanseníase 2020. Brasília: Ministério da Saúde. 2020.

BRASIL. Ministério da Saúde. Secretaria de Vigilância em Saúde. Hanseníase, verminoses e tracoma têm cura: a experiência de uma campanha integrada. Boletim Epidemiológico, Brasília, v. 47, n. 21, 2016.

BRASIL. Presidência da República. Casa Civil. Subchefia para assuntos jurídicos. Decreto ${ }^{\circ}$ 6.286, de 5 de dezembro de 2007. Brasília, 2007. Disponível em: http://www.planalto.gov.br/ccivil_03/_ato2007-2010/2007/decreto/d6286.htm. Acesso em: 20 de fevereiro de 2021.

BRITTEN, N. Entrevistas qualitativas na pesquisa em atenção à saúde. In: Pope, C.; Mays, N. Pesquisa qualitativa na atenção à saúde. 2 ed. Porto Alegre: Artmed, 2005. p. 21 - 31.

BUSS, P. M.; ARAUJO, Z. M.; PINTO, L. F.; ROCHA, C. M. F. Promoção da saúde e qualidade de vida: uma perspectiva histórica ao longo dos últimos 40 anos (1980-2020). Ciência e Saúde Coletiva, v. 25, n.12, 2020.

CASEMIRO, J. P.; FONSECA, A. B. C.; SECCO, F. V. M. Promover saúde na escola: reflexões a partir de uma revisão sobre saúde escolar na América Latina. Ciência e Saúde Coletiva, Rio de Janeiro, v. 19, n. 3, 2014.

COSTA, M. F. B. N. A.; CIOSAK, S. I.; BERTOLOMÉ, A. G.; ISERN, M. T. I. La integralidad en la atención primaria de salud en los sistemas de salud brasileño y español. Atención Primaria, v. 41, n. 7, 2009.

INSTITUTO BRASILEIRO DE GEOGRAFIA E ESTATISTICA (IBGE). Pesquisa Nacional por Amostra de Domicílios Contínua. Brasília: IBGE. 2020.

JUNQUIERA, L. A. P. A gestão intersetorial das políticas sociais e o terceiro setor. Saúde e Sociedade, v. 13, n. 1, 2004.

KELLY-SANTOS, A.; MONTEIRO, S. S.; ROSEMBERG, B. Significados e usos de materiais educativos sobre hanseníase segundo profissionais de saúde pública do Municípiodo Rio de Janeiro. Cadernos de Saúde Pública, Rio de Janeiro, v.25, n.4, p.857-67, 2009.

LEFEVRE, F.; LEFEVRE, A. M. C.; Promoção da Saúde: a negação da negação. Rio de Janeiro: Vieira e Lent, 2004.

LOPES, I. E.; NOGUEIRA, J. A. D.; ROCHA, D. G. Eixos de ação do Programa Saúde na Escola e Promoção da Saúde: revisão integrativa. Saúde Debate, v. 42, n. 118, 2018.

MATTOS, R. A. Os sentidos da integralidade: algumas reflexões acerca de valores que merecem ser defendidos. In: Pinheiro, R. e Mattos, R. A. (org). Os sentidos da integralidade na atenção e no cuidado à saúde. 8 ed. Rio de Janeiro: CEPESC, IMS/UERJ,ABRASCO, 2009. p. 43-68.

PAPOULA, S. R. O processo de trabalho intersetorial das equipes de Saúde da Famíliano município de Petrópolis-RJ: fatores restritivos e facilitadores. 2006. 186f. Dissertação (Mestrado em Saúde Pública). Programa de Pós-Graduação em Saúde Pública, Escola Nacional de Saúde Pública, Fundação Oswaldo Cruz, Rio de Janeiro, 2006.

ROCHA, P. R.; DAVID, H. M. S. Determinação ou Determinantes? Uma discussão com base na Teoria da Produção Social da Saúde. Revista da Escola de Enfermagem da USP,v. 49, n.1, 2015.

ROSEMBERG, B.; SILVA, A. P. P.; VASCONCELLOS-SILVA, P. R. Impressos hospitalares e a dinâmica de construção de seus sentidos: o ponto de vista dos profissionaisde 
saúde. Cadernos de Saúde Pública, Rio de Janeiro, v.18, n.6, p. 1685-94, 2002.

SPINK, M. J; MENEGON, V. M.; MEDRADO, B. Oficinas como estratégias de pesquisa: articulações teórico metodológicas e aplicações ético políticas. Psicologia e Sociedade, v. 26, n. 1, 2014.

VASCONCELOS, E. M. Redefinindo as práticas de Saúde a partir de experiências de Educação Popular nos serviços de saúde. Interface - Comunicação, Saúde e Educação, v.5, n. 8, 2001.

WORLD HEALTH ORGANIZATION (WHO). Integrating neglected tropical diseases into global health and development: fourth WHO report on neglected tropical diseases. Genebra: WHO. 2017. 